\title{
Si II LINES IN THE SHELL OF $\zeta$ TAU
}

\author{
SARA R. HEAP
}

Laboratory for Optical Astronomy, Goddard Space Flight Center, Greenbelt, Md., U.S.A.

\begin{abstract}
Non-LTE strengths of Si II lines were computed in an effort to reproduce the observed strengths of $\mathrm{Si}$ II lines in the shell spectrum of $\zeta$ Tau. This effort was unsuccessful, probably because it assumed that the shell could be represented by a finite, plane-parallel slab. The main conclusion of this study, then, is that in order to understand the shell spectra of Be stars, a more realistic geometry must be assumed, in which the shell is represented by a thick disk.
\end{abstract}

The availability of simultaneous ultraviolet and visual spectra of B-shell stars has made it possible for the first time to observe and measure shell lines resulting from all the important transitions of an ion. An example of these fortunate circumstances is the ion, $\mathrm{Si}$ II. Figure 1 shows all the important transitions of $\mathrm{Si}$ II. They include the

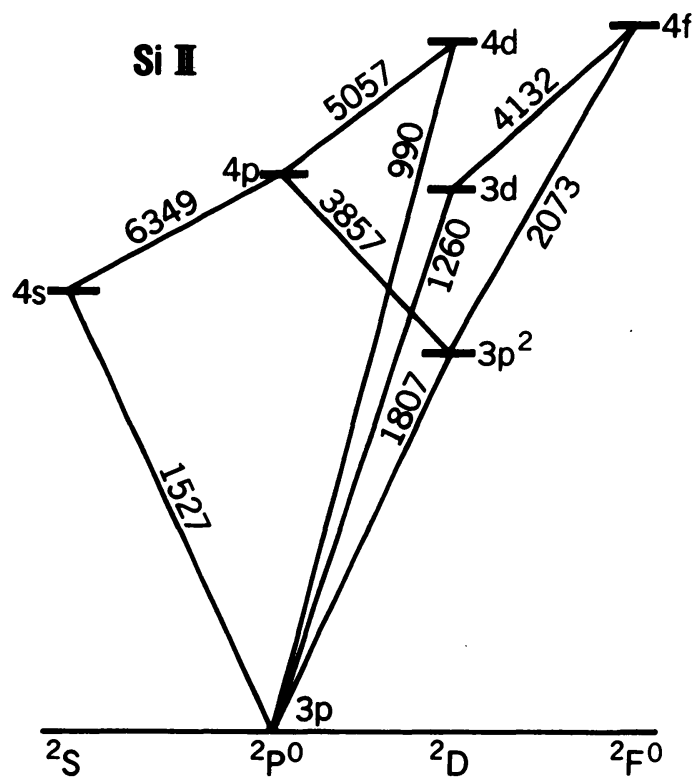

Fig. 1. Important transitions of the ion, Si II. The wavelength of each transition is indicated.

resonance lines, which all lie in the ultraviolet, and lines from excited states, most of which lie in the visual region of the spectrum. All these lines, with two exceptions, were observed in the shell spectrum of $\zeta$ Tau. The two exceptions are the $\lambda 990 \AA$ line and the $\lambda 2073 \AA$ line, both of which are beyond the range of our spectrograms. The ultraviolet spectrograms of $\zeta$ Tau were obtained in November 1972 by a rocket experiment (cf. Heap, 1975), while the visual spectrograms were obtained by Helmut Abt at the coudé focus of the 84-in. telescope at Kitt Peak National Observatory, just one night before the rocket launch. Figure 2 shows the profiles of some of these Si II 

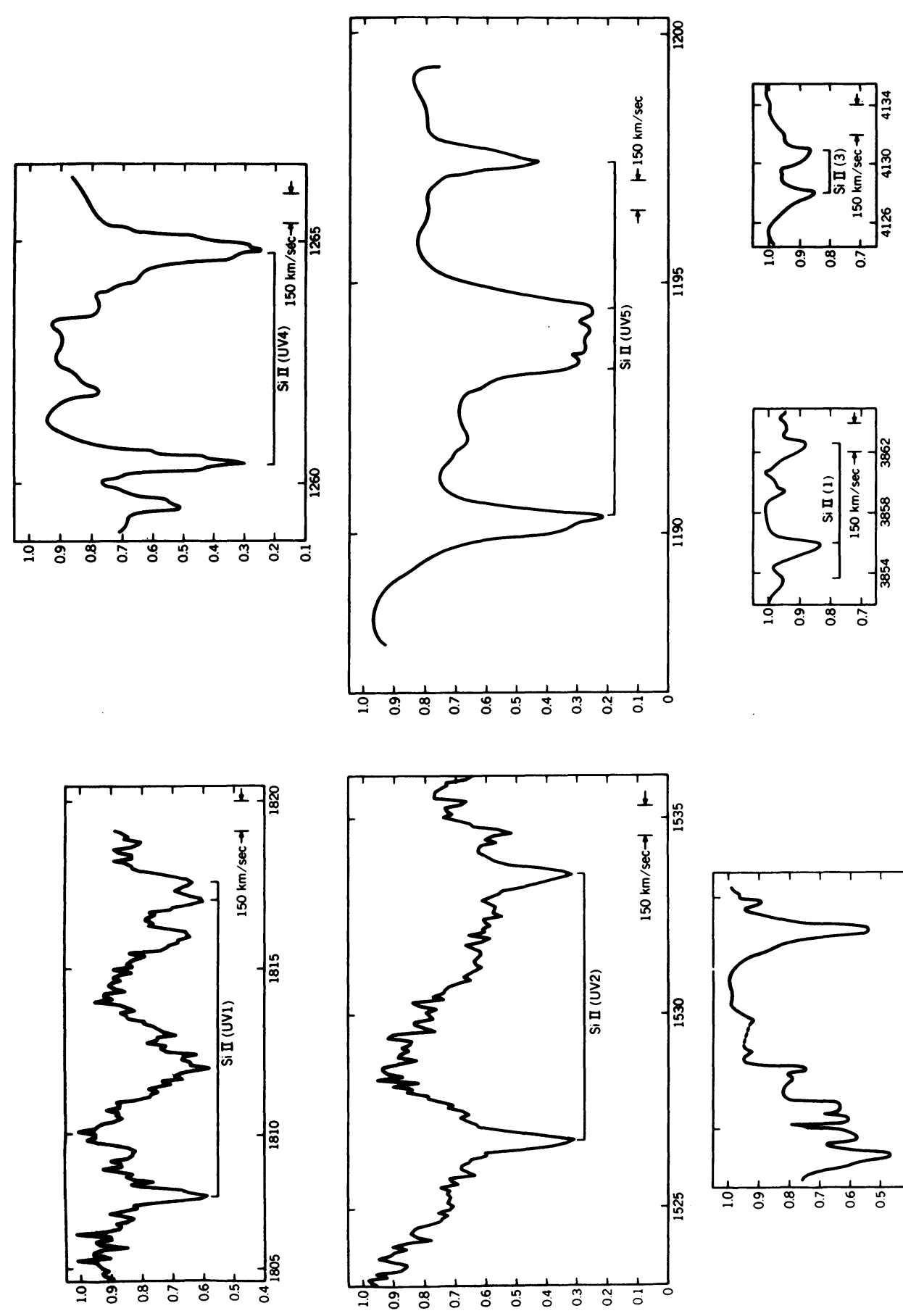

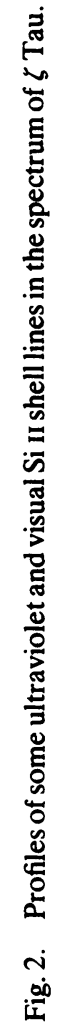


shell lines. The most important feature to note is that all the shell lines are in absorption.

I was curious to find out if these shell lines could be reproduced theoretically on the assumption that dilute radiation from the exciting star is incident on the shell, which is represented by a finite, plane-parallel slab. In order to estimate the non-LTE strengths of the Si II shell lines, I used a modified version of Kamp's (1975) non-LTE code, DRIVER. Table I gives the assumed parameters for the shell and exciting star.

TABLE I

SHELL PROPERTIES

- $\mathrm{N}_{\mathrm{e}}=1.23 \times 10^{11} \mathrm{~cm}^{-3}$

- $T_{e}=9457^{\circ} \mathrm{K}$

- COLUMN DENSITY $=0.67 \mathrm{gm} \mathrm{cm}^{-2}$

- HYDROGEN AND HELIUM ARE IN LTE

- STATIC SHELL

\section{PROPERTIES OF INCIDENT RADIATION}

- STELLAR PARAMETERS: $T_{\text {eff }}=27,500^{\circ} \mathrm{K}$, LOG $g=4.0$

- DILUTION FACTOR=25X

- SPECTRUM: PURELY CONTINUOUS (NO LINES)

The adopted value of the electron density is compatible with that obtained from the Inglis-Teller relationship as applied to the Balmer shell lines. An electron temperature of about $10000 \mathrm{~K}$ is assumed by most studies of the shell around $\zeta$ Tau, but the temperature is of little importance because at an electron density of $10^{11} \mathrm{~cm}^{-3}$, collisional transitions are very rare compared to radiative transitions. The LTE population of the $n=2$ level of hydrogen along with the total column density given in the table yields a number column density of the $n=2$ level of hydrogen of $4.9 \times 10^{15} \mathrm{~cm}^{-2}$, a value which is compatible with that derived from the observed strengths of the higher members of the Balmer series. The shell was assumed to be static because the radial velocity of the $H$ Balmer shell lines was constant with increasing upper quantum number, and hence with decreasing $g f$-value, and therefore, increasing depth of formation in the shell. The shell was assumed to lie five stellar radii away from the exciting star, which emits purely continuous radiation, characterized by the parameters, $T_{\text {eff }}=27500^{\circ}$, and $\log g=4.0$. This assumption of continuous radiation, even at the wavelengths of the Si II lines, is probably a good approximation (with one exception) since the stellar Si II lines must be very weak. The one exception is the $\mathrm{Si}$ II $\lambda 990 \AA$ line. Very probably, the $\mathrm{N}$ III line is blueshifted so that the stellar radiation at Si II $\lambda 990 \AA$ is smaller than estimated here. The boundary conditions for the equation of transfer of radiation are: (1) radiation from the star impinges normally on the inner side of the shell, and (2) no radiation 
from outside the shell impinges on the outer edge of the shell. The transfer of radiation, subject to these two boundary conditions, was solved via Feautrier's method (cf. Mihalas, 1970). The model silicon atom consisted of 13 levels of Si II and the lowest 3 levels of Si III. A bound-free transition(s) from each Si II level and 25 line transitions were taken into account. The equivalent two-level-atom approach (cf. Mihalas, 1970) was used to estimate the line source function.

The results of the computation were that the resonance lines and a few lines from excited levels were in absorption, as observed, but other lines from excited levels were in emission, contrary to observation. By looking at the net rates of the various transitions, we can see why some of the visual lines were computed to be in emission. Figure 3 shows the net rates between various levels for the mass point, $m=0.05$, a

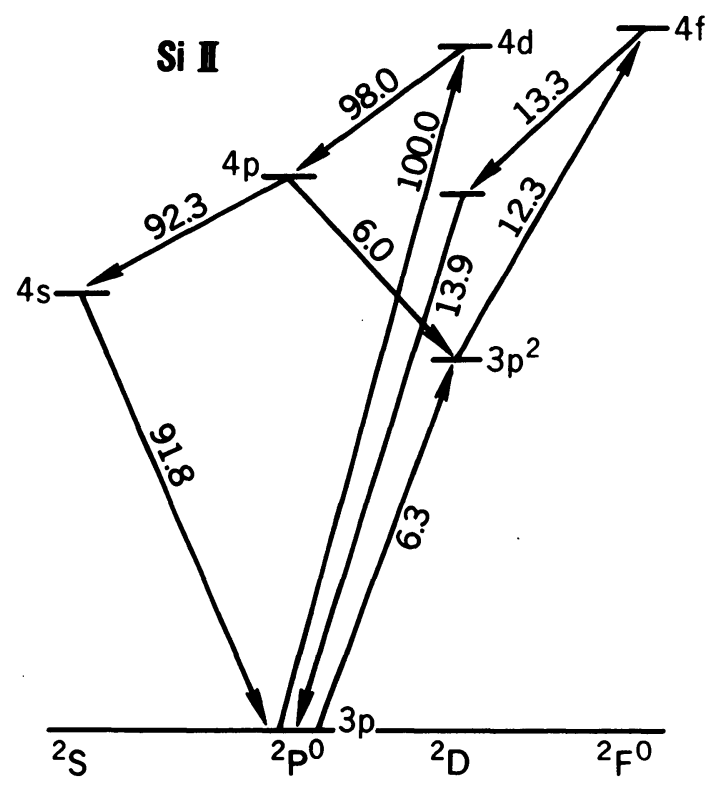

Fig. 3. Net rates between various excitation states of Si II.

layer at which the optical depth of some of the weak visual lines is about unity. The net rates in Figure 3 are normalized arbitrarily so that the strongest net rate is equal to 100 . The figure shows a pattern familiar in non-LTE physics: the existence of loops or 'Rosseland cycles'. Two are present here: one powered by the $\lambda 990 \AA$ transition, and the other powered by the $\lambda 1808 \AA$ transition. In order to stop the excited lines from going into emission, we have to stop these cycles from forming, which in effect means that we have to stop the strong net upward rates of the resonance lines. How can this be done?

An examination of the model shows two internal contradictions. The first is that the geometrical thickness of the shell is about $10^{13} \mathrm{~cm}$, so spherical symmetry should be taken into account. The second contradiction, which was not apparent until after the calculations were made, is that the mean distance before destruction of a Si II line photon is greater than the probable height of the shell, which is estimated to be about 


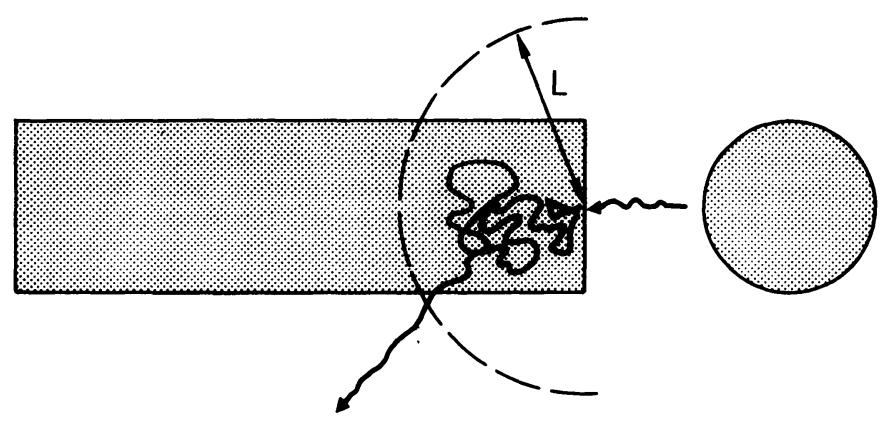

Fig. 4. Scattering of a Si II photon by the shell. The two shaded regions represent the cross-sections of the star (right) and the shell (left). See text for details.

one stellar diameter, or about $5 \times 10^{11} \mathrm{~cm}$. Figure 4 illustrates the situation. The computations show that an incoming photon from the star will be scattered many times before it is destroyed. In the process of scattering, the photon will travel a distance, $L$, given by the product of the mean-free path per scattering and the square root of the number of scatterings per destruction. For the $\mathrm{Si}$ II resonance lines, this distance is near $10^{12} \mathrm{~cm}$. The net result is that a resonance-line photon will be scattered to either the upper or lower edge of the shell and escape the shell, and therefore, not be available to drive the Rosseland cycle.

This means that the reason why all the $\mathrm{Si}$ il lines in the shell spectrum of $\zeta$ Tau appear in absorption is probably that they escaped out the upper and lower boundaries of the shell. The distinct possibility of escape from the upper and lower boundaries of the shell violates the plane-parallel assumption, which implies, first, that the thickness of the shell is small with respect to its distance from the star, but more importantly, that the shell is infinite in the directions perpendicular to incident radiation from the star. Thus there is an internal contradiction in our approach. The main conclusion, then, is that in order to understand the shell spectrum of $\zeta$ Tau, the assumption of a plane-parallel shell must be discarded, not in favor of the assumption of spherical symmetry, but rather that of cylindrical symmetry, in which the shell is represented by a thick disk.

\section{References}

Heap, S. R.: 1975, Phil. Trans. Roy. Soc. London A. 279, 371.

Mihalas, D. M.: 1970, Stellar Atmospheres, W. H. Freeman, San Francisco.

\section{DISCUSSION}

Plavec: I think this is an example of a problem which might be solved very well by the Monte Carlo method.

Poeckert: In the case of pole-on Be stars, would you expect that these lines would be in emission? Since you are allowing photons to escape below and above the plane, is that something to look for?

Heap: I don't know. 
Peters: No, these lines are not observed to be in emission in pole-on stars.

Conti: I didn't quite understand why the shell had to be more or less a donut instead of having spherical symmetry.

Heap: A thick disk provides a large surface from which the photons can escape.

Conti: I am still worried about the effect of the observed wind on the shell. Surely the wind cannot begin outside the shell unless the radius is five stellar radii and the dilution factor $W=25$. The wind must be inside the shell. How can the shell remain stable in the presence of this wind? Or is the shell maintained by the wind?

Heap: I can't tell you in physical terms; I can only tell you in observational terms: I see no evidence for any effect (e.g. heating) of the stellar wind on the shell.

Hummer: Since the asymmetric profiles of the absorption lines indicate that there is radial motion (at least) in the shell, it may be that the missing ingredient in your model is the velocity-induced photon escape.

Limber: Were the centers of the shell lines displaced?

Heap: At the time of the rocket observations, the radial velocity of the Balmer shell lines was about $20 \mathrm{~km} \mathrm{~s}^{-1}$ greater than that of the Balmer photospheric lines. 